\title{
Period-doubling bifurcations of the thermocapillary convection in a floating half zone
}

\author{
AA Yan, LI Kai, TANG ZeMei, CAO ZhongHua \& HU WenRui* \\ National Microgravity Laboratory, Institute of Mechanics, Chinese Academy of Sciences, Beijing 100190, China
}

Received May 25, 2010; accepted July 8, 2010; published online July 21, 2010

\begin{abstract}
This study experimentally explored the fine structures of the successive period-doubling bifurcations of the time-dependent thermocapillary convection in a floating half zone of $10 \mathrm{cSt}$ silicone oil with the diameter $d_{0}=3.00 \mathrm{~mm}$ and the aspect ratio $A=l / d_{0}=0.72$ in terrestrial conditions. The onset of time-dependent thermocapillary convection predominated in this experimental configuration and its subsequent evolution were experimentally detected through the local temperature measurements. The experimental results revealed a sequence of period-doubling bifurcations of the time-dependent thermocapillary convection, similar in some way to one of the routes to chaos for buoyant natural convection. The critical frequencies and the corresponding fractal frequencies were extracted through the real-time analysis of the frequency spectra by Fast-Fourier-Transformation (FFT). The projections of the trajectory onto the reconstructed phase-space were also provided. Furthermore, the experimentally predicted Feigenbaum constants were quite close to the theoretical asymptotic value of 4.669 [Feigenbaum M J. Phys Lett A, 1979, 74: 375-378].
\end{abstract}

thermocapillary convection, bifurcation, transition to turbulence

PACS: $47.55 . n b, 47.20 . \mathrm{Ky}, 47.27 . \mathrm{Cn}$

\section{Introduction}

The transition of fluid convection to chaos in dissipative dynamical systems is theoretically and practically interesting in fluid mechanics. The routes to chaos for a buoyant natural convection have attracted extensive studies (see [1-9]). The studies have shown several typical appearances that might announce chaos, such as period-doubling phenomenon [6], the Ruelle-Takens-Newhouse route [7,8] and the intermittent transition [9], depending on the controlling parameters of the dissipative dynamical systems such as the Rayleigh number, the Prandtl number and the geometric aspects. Another important type of natural convection is the thermocapillary convection driven by the surface-tension gradient in fluid systems with an interface subject to microgravity conditions or in small-scale terrestrial configurations.

\footnotetext{
*Corresponding author (email: wrhu@imech.ac.cn)
}

In the latter case, the relative importance of the gravity effect to the capillary effect is scaled by the dynamic Bond number $B_{d}=\rho \beta g l^{2} /\left|\sigma_{T}^{\prime}\right|$, where $g=9.8 \mathrm{~m} \mathrm{~s}^{-2}$ is the earth gravity, $l$, the geometry scale of the configuration, and $\rho, \beta$ and $\sigma_{T}$, the density, the thermal expansion coefficient and the temperature gradient of the surface tension of the fluid respectively. The geometric scale of the configuration in terrestrial conditions, therefore, is significantly reduced to highlight the capillary effect. As a new dissipative dynamical system, thermocapillary convection has become one of the fundamental subjects in microgravity fluid physics and space fluid/heat management [10]. A floating half zone consisting of a liquid column confined between differentially heated solid rods, as shown in Figure 1, was initially introduced to mimic half of the floating zone technique which is of interest to space materials science, and has now become one of the typical models for investigation into the principles of thermocapillary convection. Originated by 


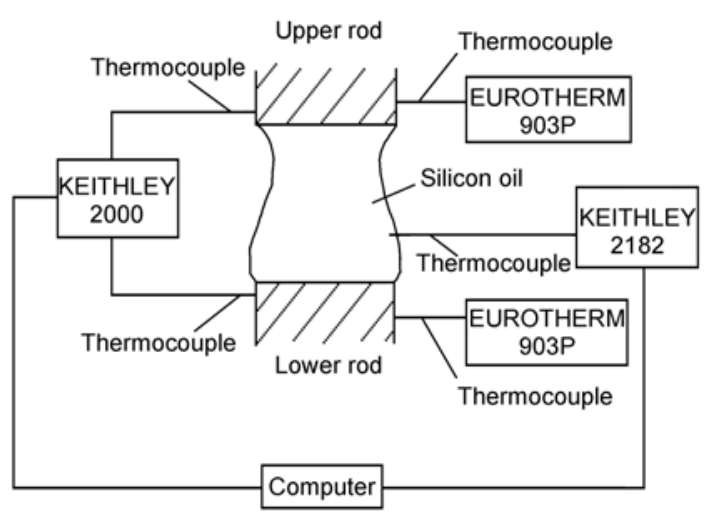

Figure 1 Schematic sketch of the experimental apparatus.

Chun and Wuest $[11,12]$ and Schwabe et al. $[13,14]$ respectively, extensive theoretical and experimental studies have been conducted on the thermocapillary convection in a floating half zone in microgravity or terrestrial conditions (refer to the review work in [10]). The studies revealed that with an increasing applied temperature difference between the supporting rods, the thermocapillary convection changes from a steady convection to a time-dependent convection and then to the turbulent convection. However, most studies focused on the onset of time-dependent thermocapillary convection, the very initial regime of the route to chaos. The exploration of possible routes to chaos for the thermocapillary convection in a floating half zone is still an attractive open question due to its strong non-linear characteristics, especially in experimental research. It is worth noting that the quasi-periodic flow state, the period-doubling phenomenon, the spatial-temporal intermittency and the $\mathrm{Ru}$ elle-Takens-Newhouse route, which are similar to those in the buoyant natural convection, were experimentally reported in refs. [15-19] to some extent.

In the present study, the time-dependent thermocapillary convection predominated in a floating half zone of $10 \mathrm{cSt}$ silicone oil has been experimentally investigated in terrestrial conditions. The fine structures of the successive period-doubling bifurcations of the thermocapillary convection were reported through local temperature measurements in the liquid column. The corresponding critical conditions were predicted. Furthermore, the Feigenbaum constant was experimentally measured and compared with the theoretical prediction.

\section{Experimental apparatus and procedures}

As shown in Figure 1, a floating half zone of $10 \mathrm{cSt}$ silicone oil formed between two co-axial rods of the same diameter $\left(d_{0}=3.00 \mathrm{~mm}\right)$. A temperature difference was gradually applied at a certain heating rate between the supporting rods, and the lower rod was kept at the constant temperature. Two PID-controllers (Eurotherm 904 Controller) were used to control the heating rate that enabled the applied temperature difference measured by two thermocouples of T-type (see Figure 1) to have an accuracy better than $0.05 \mathrm{~K}$. For the reduction of disturbance on the flow state, only one rather small thermocouple of the K-type $(0.02 \mathrm{~mm}$ in diameter) was inserted into the liquid column at $0.35 l$ above the lower rod and right beneath the free surface, and the corresponding local temperature change was registered by a Keithely 2182 with a resolution of $0.001 \mathrm{~K}$. The non-contact temperature measurement was also attempted, i.e., the thermocouples were placed as close to the free surface as possible, but without any physical contact with it. However, the corresponding SNR (Signal to Noise Ratio) and the reproducibility of the experiments were not ideal. Another two thermocouples of the T-type were attached to the rods in the vicinity of the rod/liquid interface, and the corresponding temperature signals were registered by a multimeter Keithely 2000 with a resolution of $0.01 \mathrm{~K}$.

It is crucial to experimentally determine the exact onset of the time-dependent thermocapillary convection and the sequenced bifurcations. In the present study, preliminary experimental studies were conducted with relatively fast heating rates to establish the approximate critical temperature differences corresponding to the bifurcations. In the main experiment, the temperature difference controlled by the PID-controllers was increased at a heating rate of 0.05 $\mathrm{K} / \mathrm{s}$ to values in the vicinity of the pre-determined thresholds (about $2 \mathrm{~K}$ below) and was held for several minutes. Then the temperature difference was further increased at different time points until the occurrence of the bifurcation. At each step, the temperature difference was increased at a much lower heating rate of $0.005 \mathrm{~K} / \mathrm{s}$ to the expected value and held for 5-8 min to allow the convection to fully develop. In the small-scale configuration used, the timedependent thermocapillary convection with a frequency of several $\mathrm{Hz}$ arose, which allowed fast data-sampling and relatively short experiment duration. A scanning rate of 10 $\mathrm{Hz}$ was adopted as a compromise to the measurability limit of the fine structures of the thermocapillary convection in the present study. The temperature measurement signals were analyzed in a frequency domain to predict the critical frequencies and corresponding fractal frequencies through the Fast-Fourier-Transformation (FFT) in real time where the cut-off filter is applied with automatically adjusted cut-off frequency and steepness.

The volume ratio $V / V_{0}$ is another sensitive critical parameter [10], where $V$ is the volume of the liquid column and $V_{0}$ is the volume of a cylindrical column with the same aspect ratio. The curvature variation in the longitudinal direction of the free surface alters the equilibrium of the forces and then the onset of time-dependent thermocapillary convection. In the present study, the volume of the floating half zone can be obtained through a measurement system consisting of a CCD camera and a PC with an image-acquiring card. The boundaries of the floating half zone were 
defined by processing its images, and then the volume of floating half zone can be calculated. The injection of the silicon oil before the onset of the time-dependent convection allows the compensation of the volume change due to the thermal expansion and evaporation. The uncertainty in the determination of the Marangoni number in the present study is $\pm\left(0.01 M a_{c}+2 M a_{c} / \Delta T_{c}\right)$ where the former is from the measurement of the geometry of the floating half zone and the latter is from the measurement of the critical temperature difference.

\section{Experimental results}

For the floating half zone with a diameter of $d_{0}=3.00 \mathrm{~mm}$ and an aspect ratio of $A=l / d_{0}=0.72$ of the $10 \mathrm{cSt}$ silicone oil analyzed in the present study, the dynamic Bond number is $B_{d}=\rho \beta g l^{2} /\left|\sigma_{T}^{\prime}\right|=0.77$ (see Table 1). Therefore, the thermocapillary convection predominates in the floating half zone even in the terrestrial conditions. Based on our experimental studies (not all reported here), the aspect ratio and the volume ratio of the floating half zone are both crucial for determining the route to chaos of the thermocapillary convection. The floating half zone of the aspect ratio $l / d_{0}=0.72$ and the volume ratio $V / V_{0}=0.63$ is adopted in the present study in which a typical successive period-doubling phenomenon occurs.

Table 1 Thermo-physical properties of $10 \mathrm{cSt}$ silicone oil

\begin{tabular}{cc}
\hline$\rho$ & $935\left(\mathrm{~kg} \mathrm{~m}^{-3}\right)$ \\
\hline$\beta$ & $1.08 \times 10^{-3}\left(\mathrm{~K}^{-1}\right)$ \\
$\sigma_{T}$ & $6.0 \times 10^{-5}\left(\mathrm{~kg} \mathrm{~K}^{-1} \mathrm{~S}^{-2}\right)$ \\
\hline
\end{tabular}

In the experiment, the applied temperature difference controlled by the PID-controllers is gradually increased. When the temperature difference is increased to $\Delta T=$ $(45.07 \pm 0.05) \mathrm{K}$, indicated by the temperature measurements through the thermocouple in the liquid column, the temperature field starts to fluctuate periodically at a single oscillation frequency of $f_{0}=1.378 \mathrm{~Hz}$ (see the corresponding FFT spectrum in Figure 2(a)). Right after the Hopf bifurcation, the temperature difference is held for several minutes to allow the full development of the time-dependent thermocapillary convection (see Figure 2(b)). When the temperature difference is increased to $\Delta T=(91.99 \pm 0.05) \mathrm{K}$, the first period-doubling bifurcation occurs. At this moment, the main oscillation frequency shifts to $f_{0}=2.28 \mathrm{~Hz}$ with the fractal frequency $f_{1 / 2}$ locked at exactly $f_{0} / 2=1.14 \mathrm{~Hz}$ (see Figure 3(a)). The period-doubling bifurcation can be clearly observed in the fully developed temperature oscillation as shown in Figure $3 \mathrm{~b}$. When the temperature difference is increased to $\Delta T=(102.06 \pm 0.05) \mathrm{K}$, the second period-doubling bifurcation occurs with $f_{1 / 4}=f_{0} / 4=0.67 \mathrm{~Hz}$ while the
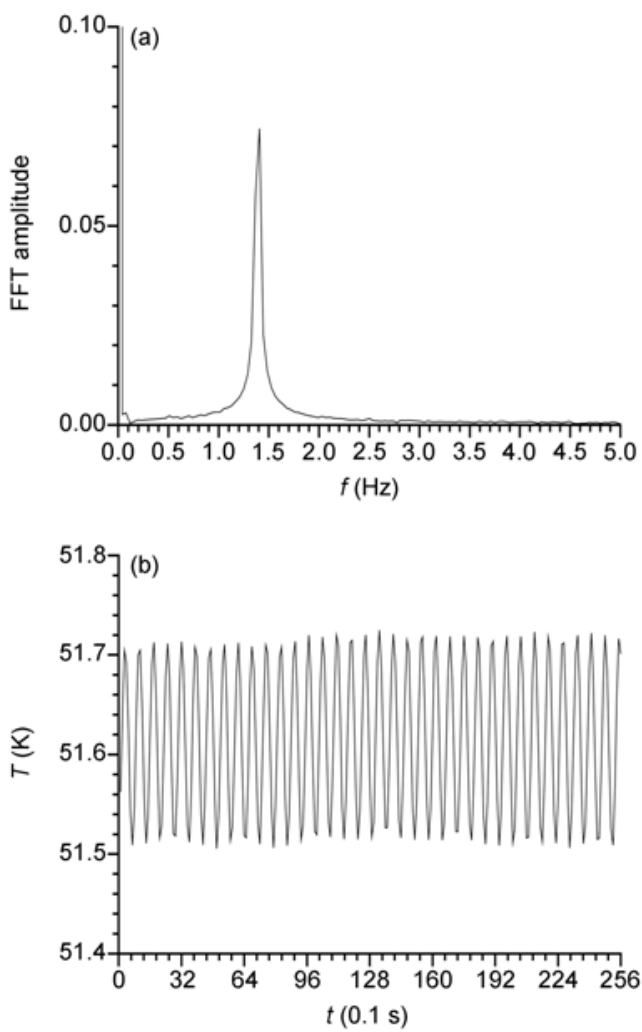

Figure 2 The temperature evolution (a) and the corresponding frequency spectrum through the thermocouple in the liquid column at the onset of oscillation (b).
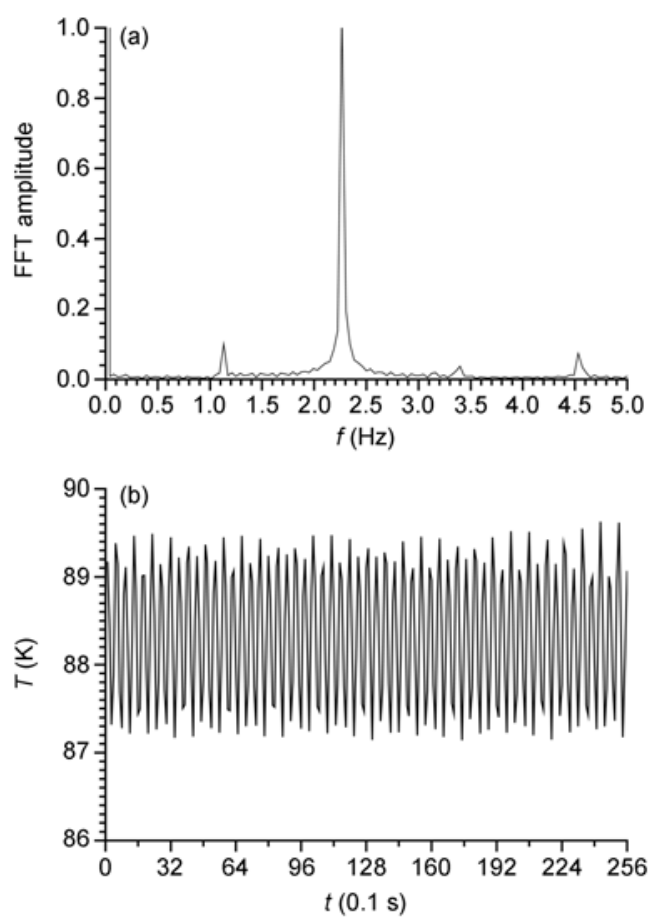

Figure 3 The temperature evolution (a) and the corresponding frequency spectrum through the thermocouple in the liquid column when $f_{1 / 2}$ occurs (b). 
main oscillation frequency shifts to $f_{0}=2.68 \mathrm{~Hz}$ (see Figure 4). The other fractal frequencies, $f=2 f_{0} / 4=1.34 \mathrm{~Hz}$ and $f=3 f_{0} / 4=2.01 \mathrm{~Hz}$, also appear in the spectrum. When the temperature difference is increased to $\Delta T=(104.35 \pm 0.05) \mathrm{K}$, the third period-doubling bifurcation occurs with $f_{1 / 8}=f_{0} / 8=$ $0.35 \mathrm{~Hz}$ while the main oscillation frequency slightly shifts to $f_{0}=2.76 \mathrm{~Hz}$ (see Figure 5). The other fractal frequencies, except for $f=2 f_{0} / 8$ and $f=5 f_{0} / 8$, also appear in the spectrum. Note that when the temperature difference is slightly increased, i.e., right after the third period-doubling bifurcation, the experiment cannot be continued due to the breakage of the liquid column. In all the attempts in our experimental study, the regime of chaos could not be observed in the present small-scale terrestrial configuration where the thermocapillary convection predominated.

In representing the original periodic orbit and the orbits for three successive period-doubling bifurcations, a 3D phase-space based on the time-delay was reconstructed [20]. The embedding dimension is $m=3$, and the time delay $\tau$ is selected to make the attractor to be unfolded in this demension. Figure 6 shows the projections of the trajectory onto the reconstructed phase-space where the axes correspond to $T(t), T(t+\tau)$ and $T(t+2 \tau)$. A limit cycle can be clearly observed in Figure 6(a) which represents the periodic nature of the thermocapillary convection at a unique frequency, and the deformed shape of the attractor in Figures 6(c) and (d) is attributed to the successive period-doubling bifurcations.
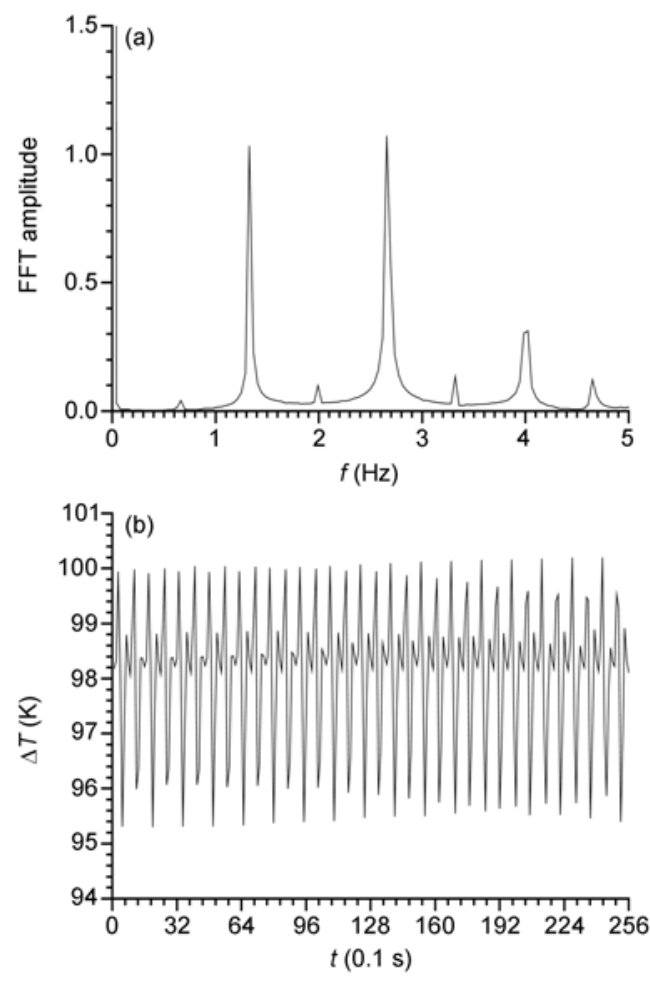

Figure 4 The temperature evolution (a) and the corresponding frequency spectrum through the thermocouple in the liquid column when $f_{1 / 4}$ occurs (b).
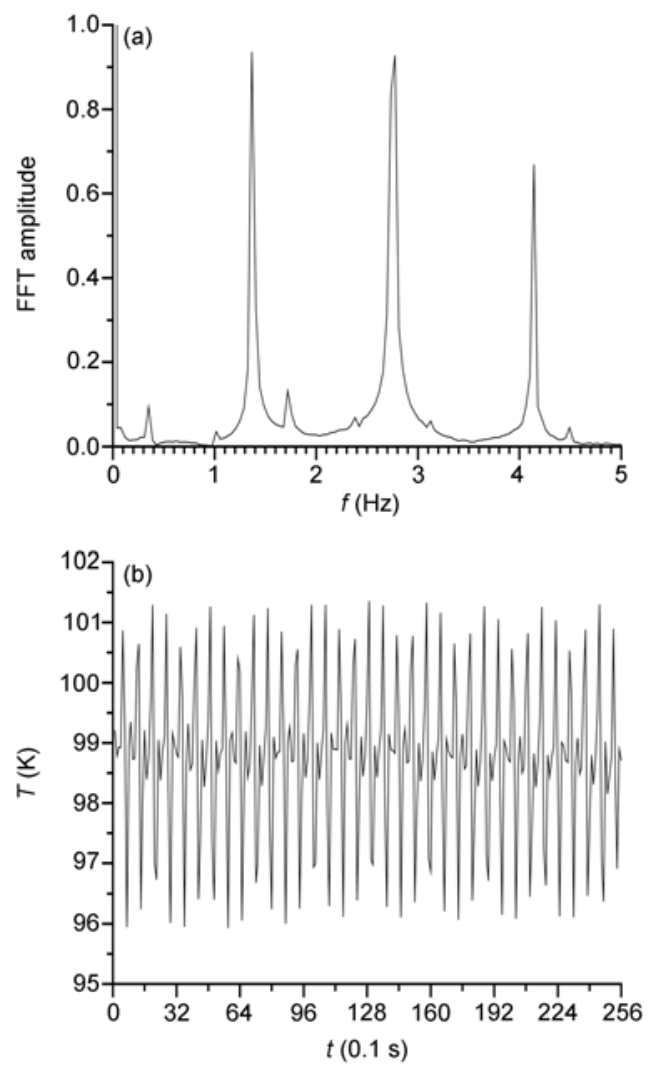

Figure 5 The temperature evolution (a) and the corresponding frequency spectrum through the thermocouple in the liquid column when $f_{1 / 8}$ occurs (b).

Finally, the Feigenbaum constant was experimentally measured. According to Feigenbaum's general theory for the fluctuation spectrum of the route to chaos, value $a_{N}$ of the constraint should asymptotically approach the relation, $\delta_{N}=\left(a_{N+1}-a_{N}\right) /\left(a_{N+2}-a_{N+1}\right) \rightarrow \delta$, where $\delta=4.669$. In the present study, the Feigenbaum constants calculated based on the critical temperature differences for the successive period-doubling bifurcations were respectively

$$
\delta_{e 1}=(91.99-45.07) /(102.06-91.99)=4.660
$$

and

$$
\delta_{e 2}=(102.06-91.99) /(104.35-102.06)=4.400,
$$

which are quite close to the universal constant predicted by Feigenbaum's general theory [1].

\section{Conclusions}

Usually the fluid convection in a floating half zone in terrestrial conditions is a mixture of thermocapillary convection driven by the non-uniformity of free surface tension and the Benard convection driven by the buoyancy. However, in a floating half zone of $10 \mathrm{cSt}$ silicone oil $\left(d_{0}=3.00\right.$ 


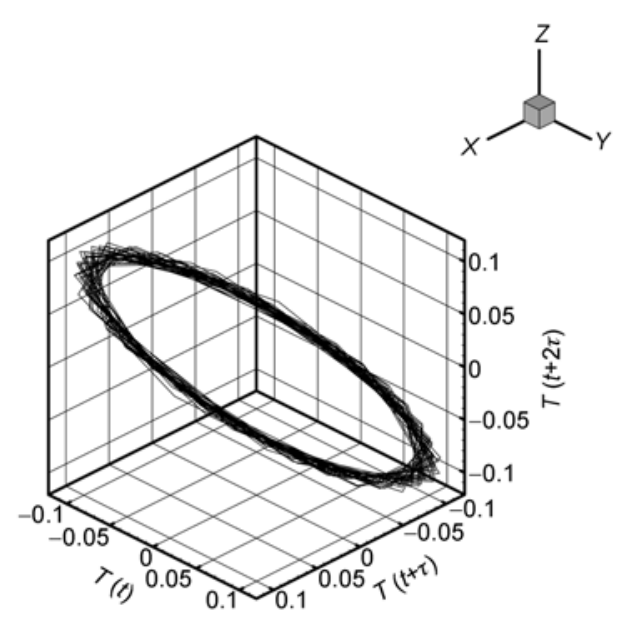

(a)

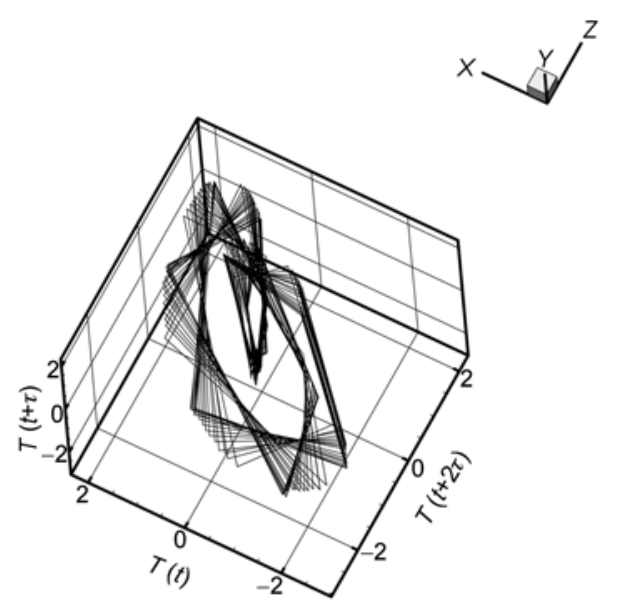

(c)

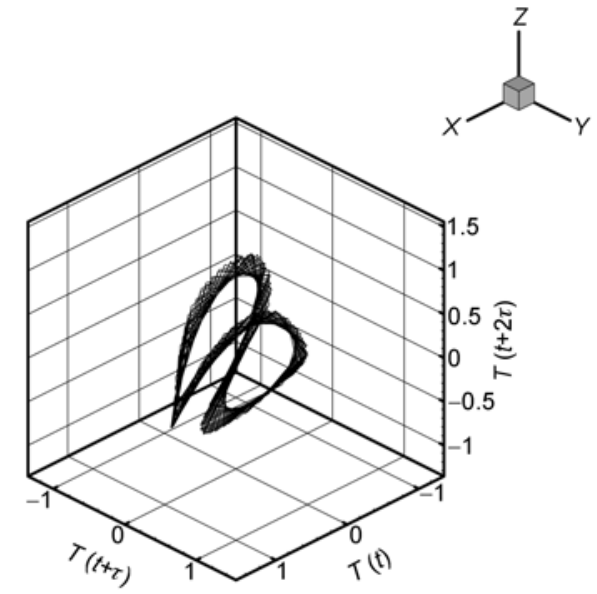

(b)

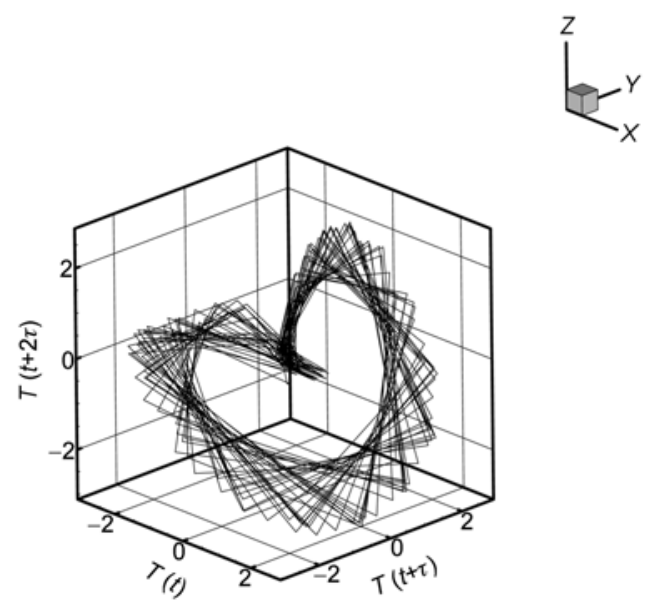

(d)

Figure $63 \mathrm{D}$ time-delay representation of the temperature through the thermocouple in the liquid column. (a) $\Delta T=(45.07 \pm 0.05) \mathrm{K}$; (b) $\Delta T=(91.99 \pm 0.05) \mathrm{K}$; (c) $\Delta T=(102.66 \pm 0.05) \mathrm{K}$; (d) $\Delta T=(104.35 \pm 0.05) \mathrm{K}$.

$\mathrm{mm}, A=l / d_{0}=0.72$ and $\left.V / V_{0}=0.63\right)$ as adopted in the present experimental study, the thermocapillary convection predominates due to the small dynamic Bond number $B_{d}=0.77$. Compared to the studies on buoyant natural convection, the investigation into the routes to chaos for the thermocapillary convection is insufficient, especially from the perspective of empirical research.

In the current study, fine structures of the successive period-doubling bifurcations of thermocapillary convection are observed through the local temperature measurements. The corresponding fractal frequencies are predicted as $f_{1 / 2}=1.14 \mathrm{~Hz}, f_{1 / 4}=0.67 \mathrm{~Hz}$ and $f_{1 / 8}=0.35 \mathrm{~Hz}$. The experimental results show that the period-doubling phenomenon is also typical in the transition process of thermocapillary convection for the parameters discussed in the current study, and the experimental results support the idea that the timedependent thermocapillary convection in a floating half zone is induced by internal instability. Furthermore, the Feigenbaum constants experimentally measured in this study are $\delta_{e 1}=4.660$ and $\delta_{e 2}=4.400$, which are close to Feigenbaum's theoretical prediction of $\delta=4.669$.

At the initial stage of investigation, the current study indicated a transition process of period-doubling bifurcations in specific critical parameters. For a complex dissipative dynamical system of fluid mechanics, other routes to chaos for the thermocapillary convection are found in other ranges of critical parameters, and the corresponding fine structures require further insights.

This work was supported by the Knowledge Innovation Project of the Chinese Academy of Sciences (Grant No. KJCX2-YW-L08). The authors thank Profs. LIU ShiDa and WEI ZhongLei for helpful discussions.

1 Feigenbaum M J. The onset spectrum of turbulence. Phys Lett A, 1979, 74: 375-378

2 Gollub J P, Benson S V. Many routes to turbulent convection. J Fluid Mech, 1980, 100: 449-470

3 Braun R, Feudel F, Guzdar P. Route to chaos for a two-dimensional 
externally driven flow. Phys Rev E, 1998, 58: 1927-1932

4 Bucchignani E, Stella F. Rayleigh-Benard convection in domains: part 2-Transitions to chaos. Numer Heat Transfer Part A, 1999, 36: 17-34

5 Saha A K, Biswas G, Muralidhar K. Transition and chaos in twodimensional flow past a square cylinder. J Eng Mech, 2000, 126: 523-532

6 Libchaber S, Maurer J. Local probe in a Rayleigh-Benard experiment in liquid helium. J Phys Lett, 1978, 39: 369-372

7 Ruelle D, Takens F. On the nature of turbulence. Commun Math Phys, 1971, 20: 167-192

8 Newhouse S, Ruelle D, Takens F. Occurrence of strange axiom a attractors near quasiperiodic flows on $\mathrm{Tm}, \mathrm{m} \geqslant 3$. Commun Math Phys, 1978, 64: 35-40

9 Pomeau Y, Manneville P. Intermittent transition to turbulence in dissipative dynamical systems. Commun Math Phys, 1980, 74: 189-197

10 Hu W R, Tang Z M, Li K. Thermocapillary convection in floating zones. Appl Mech Rev, 2008, 61: 010803

11 Chun $\mathrm{C} \mathrm{H}$, Wuest W. A micro-gravity simulation of the Marangoni convection. Acta Astronaut, 1978, 5: 681-686

12 Chun $\mathrm{C} \mathrm{H}$, Wuest W. Experiments on the transition from the steady to the oscillatory Marangoni-convection of a floating zone under reduced gravity effect. Acta Astronaut, 1979, 6: 1073-1079
13 Schwabe D, Scharmann A, Preisser F, et al. Experiments on surface tension driven flow in floating zone melting. J Cryst Growth, 1978, 43: 305-312

14 Schwabe D, Scharmann A. Some evidence for the existence and magnitude of a critical Marangoni number in crystal growth melts. J Cryst Growth, 1979, 46: 125-131

15 Velten R, Schwabe D, Scharmann A. Gravity-dependence of the instability of surface-tension-driven flow in floating zones. In: Proc. VII $^{\text {th }}$ European Symp. on materials and fluid sciences in microgravity, ESA SP-295, 1990. 271-278

16 Tang Z M, Hu W R, Xie J C, et al. Transition from steady to oscillatory convection with chaotic feature in thermocapillary convection. Adv Space Res, 1995, 16: 67-70

17 Frank S, Schwabe D. Temporal and spatial elements of thermocapillary convection in floating zones. Exp Fluids, 1997, 23: 234-251

18 Ueno I, Tanaka S, Kawamura H. Oscillatory and chaotic thermocapillary convection in a half-zone liquid bridge. Phys Fluids, 2003, 15: 408-416

19 Schwabe D, Frank S. Experiments on the transition to chaotic thermocapillary flow in floating zones under microgravity. Adv Space Res, 1999, 24: 1391-1396

20 Baker G L, Gollub J P. Chaotic dynamics, an introduction. New York: Cambridge University Press, 1996 